\title{
AMYOTROPHIC LATERAL SCLEROSIS-SPECIFIC QUALITY OF LIFE- SHORT FORM (ALSSQOL-SF): A BRIEF, RELIABLE, AND VALID VERSION OF THE ALSSQOL-R
}

\author{
STEPHANIE H. FELGOISE, PHD, ${ }^{1}$ RICHARD FEINBERG, PHD, ${ }^{2}$ HELEN E. STEPHENS, $M S,{ }^{3}$ PAUL BARKHAUS, MD, ${ }^{4}$ \\ KEVIN BOYLAN, MD, ${ }^{5}$ JAMES CARESS, MD, ${ }^{6}$ LORA L. CLAWSON, MSN, CRNP, ${ }^{7}$ LAUREN ELMAN, MD, ${ }^{8}$ \\ STEPHEN A. GOUTMAN, MD $\mathbb{D}^{10},{ }^{9}$ LEO MCCLUSKEY, MD, ${ }^{8}$ JAMES RUSSELL, DO, ${ }^{10}$ EZGI TIRYAKI, MD, ${ }^{11}$ MICHAEL WEISS, MD, ${ }^{12}$ \\ and ZACHARY SIMMONS, MD (D) ${ }^{3}$ \\ ${ }^{1}$ Department of Psychology, Philadelphia College of Osteopathic Medicine, Philadelphia, Pennsylvania, USA \\ ${ }^{2}$ National Board of Medical Examiners, Philadelphia, Pennsylvania, USA \\ ${ }^{3}$ Department of Neurology, Penn State Hershey Medical Center, Department of Neurology, EC 037, P.O. Box 859, Hershey, Pennsylvania, \\ 17033, USA \\ ${ }^{4}$ Department of Neurology, Medical College of Wisconsin, Milwaukee, Wisconsin, USA \\ ${ }^{5}$ Department of Neurology, Mayo Clinic Jacksonville, Jacksonville, Florida, USA \\ ${ }^{6}$ Department of Neurology, Wake Forest University, Winston-Salem, North Carolina, USA \\ ${ }^{7}$ Department of Neurology, The Johns Hopkins University School of Medicine, Baltimore, Maryland, USA \\ ${ }^{8}$ Department of Neurology, University of Pennsylvania, Philadelphia, Pennsylvania, USA \\ ${ }^{9}$ Department of Neurology, University of Michigan, Ann Arbor, Michigan, USA \\ ${ }^{10}$ Department of Neurology, Lahey Clinic, Burlington, Massachusetts, USA \\ ${ }^{11}$ Department of Neurology, University of Minnesota, Minneapolis, Minnesota, USA \\ ${ }^{12}$ Department of Neurology, University of Washington, Seattle, Washington, USA \\ Accepted 18 June 2018
}

ABSTRACT: Introduction: The Amyotrophic Lateral Sclerosis (ALS)-Specific Quality of Life instrument and its revised version (ALSSQOL and ALSSQOL-R) have strong psychometric properties, and have demonstrated research and clinical utility. In this study we aimed to develop a short form (ALSSQOL-SF) suitable for limited clinic time and patient stamina. Methods: The ALSSQOL-SF was created using Item Response Theory and confirmatory factor analysis on 389 patients. A cross-validation sample of 162 patients assessed convergent, divergent, and construct validity of the ALSSQOL-SF compared with psychosocial and physical functioning measures. Results: The ALSSQOL-SF consisted of 20 items. Compared with the ALSSQOL-R, optimal precision was retained, and completion time was reduced from 15-25 minutes to 2-4 minutes. Psychometric properties for the ALSSQOL-SF and its subscales were strong. Discussion: The ALSSQOL-SF is a disease-specific global QOL instrument that has a short administration time suitable for clinical use, and can provide clinically useful, valid information about persons with ALS.

Muscle Nerve 58:646-654, 2018

Quality of life (QOL) is a broad term with many operational definitions. Health-related QOL, or health status, is most commonly used to describe an individual's perception of their well-being secondary to their physical and mental functioning. ${ }^{1,2}$ However, QOL is often conceived more broadly as being impacted by many factors other than the physical or mental health domains, such as family and friends, finances, religion and spirituality, and existential concerns. ${ }^{3}$ When defined in this manner, overall QOL in patients with amyotrophic lateral sclerosis (ALS) does not correlate with physical function and appears to be maintained over time as physical function declines. ${ }^{4-10}$ Baseline and ongoing assessments provide insight into factors that impact QOL, and they may guide multidisciplinary interventions for ALS patients. One instrument that captures this global self-perception of QOL is the 59-item ALSSpecific Quality of Life Instrument (ALSSQOL). This questionnaire, and its revised, shorter version, the ALSSQOL-R, have been validated in multicenter studies. ${ }^{11-13}$ The ALSSQOL-R is a 50-item (46 scored) instrument that measures overall QOL with 6 subscales (Negative Emotion, Interaction with People and the

Additional supporting information may be found in the online version of this article.

Key words: ALS, ALSSQOL, amyotrophic lateral sclerosis, assessment, psychometrics, quality of life

Abbreviations: ALSSQOL, ALS-Specific Quality of Life Instrument; ALSSQOL-R, ALS-Specific Quality of Life Instrument-Revised; ALSSQOL-SF, ALS-Specific Quality of Life Instrument-Short Form; BSI-18, Brief Symptom Inventory-18-item version; CES-D, Center for Epidemiological Studies-Depressed Mood Scale; CFA, confirmatory factor analysis; CFI, comparative fit index; IIR, Idler Index of Religiosity; IRT, Item Response Theory; M-GRM, Modified Graded Response Model; MQOL-SIS, McGill Quality of Life Single Item Score; QOL, quality of life; RMSEA, root-mean-square error of approximation; SRMR, standard root-meansquare residual; SWLS, Satisfaction with Life Scale; WHOQOL-BREF, World Health Organization Quality of Life Instrument-Brief version Funding: Paul and Harriett Campbell Fund for ALS Research; ALS Association Greater Philadelphia Chapter

Disclosures and Conflicts of Interest: S.A.G. has received honorarium from Continuum, and has consulted for Cytokinetics. J.R. has served as a paid data monitoring committee member for Cytokinetics, received royalties for publication from McGraw-Hill, been paid to review medical records related to medical-legal proceedings, and has been a paid participant in ALS marketing research surveys. M.W. has been a speaker for Soleo Health. Z.S. has served as a paid consultant for Cytokinetics and Neuralstem. He serves as Editor-in-Chief of Muscle \& Nerve, but played no role in the review of this manuscript or in decisions regarding its acceptance or rejection, which were managed entirely by an associate editor.

Correspondence to: Z. Simmons; e-mail: zsimmons@psu.edu

(C) 2018 Wiley Periodicals, Inc.

Published online 20 July 2018 in Wiley Online Library (wileyonlinelibrary.com). DOI 10.1002/mus.26203 
Environment, Intimacy, Religiosity, Physical Symptoms, and Bulbar Function) and an average total QOL score. Every subscale consists of several items, each of which is rated by the patient on a $0-10$-point Likert-type scale $(0=$ least desirable, $10=$ most desirable). Average total QOL score is the average of all items, ranging from 0 (worst QOL) to 10 (best QOL).

Completion of the ALSSQOL-R by persons with ALS takes approximately 15-25 minutes by interview. Fatigue may make an assessment of this length highly taxing or burdensome for some patients, particularly in view of research showing that patients already cite the length of visits as a negative feature of the multidisciplinary ALS clinic. ${ }^{14}$ In addition, it may be challenging for busy ALS clinic personnel to find time to administer the questionnaire. In view of the utility of the ALSSQOL-R and these limiting factors, we sought to develop and validate a shorter version, the ALSSQOL-Short Form (ALSSQOL-SF).

\section{METHODS}

For all studies and phases, institutional review board (IRB) approval was obtained from all participating institutions, and all participants provided informed consent.

\section{Phase 1: Creation of the ALSSQOL-SF Patients. Phase} 1 was a cross-sectional study using data from a validation study of the ALSSQOL-R collected between 2005 and 2008. ${ }^{13}$ All participants had definite, probable, probable laboratory-supported, or possible ALS, ${ }^{15}$ were $\geq 18$ years of age, and fluent in English at the grade 6 level or higher. Patients were excluded if a physician or psychologist at the center judged them to have cognitive impairment sufficient to preclude the granting of informed consent and participation in the study.

Item Response Theory Methodology. Item Response Theory (IRT) analyses were conducted to inform ways of shortening the ALSSQOL-R with minimal loss of information. ${ }^{16}$ IRT is a test design, analysis, and scoring approach that utilizes statistical modeling to distinguish between the characteristics of the person from the characteristics of the items to which they responded. ${ }^{16}$ IRT is useful in test construction by using itemlevel information to identify items that best suit the purpose for a given measure. In an IRT context, information is equivalent to the concept of reliability in classical test theory, except that it can vary. In IRT, the precision of an item or measure depends on the location of the individual on the trait scale, whereas reliability makes an assumption that error is constant. Using IRT to build a measure involves defining the ideal information function and then selecting items that best approximate that shape. ${ }^{17}$ In designing a short form, the ideal information function is the information function for the original form. Thus, IRT can be effective in creating a short form that provides similar information across the trait scale.

To provide guidance about which items should be removed from the ALSSQOL-R to create the ALSSQOL-SF, item parameters for each of the 6 subscales were estimated with the Modified Graded Response Model (M-GRM) ${ }^{18}$ using PARSCALE. ${ }^{19}$ The slope parameter $(\alpha)$ is an indicator of discrimination power, the extent to which the item can distinguish between individuals with low and high trait levels, whereas the location parameter $(\beta)$ indicates the targeted trait value. ${ }^{20}$ Items were selected based on: (i) large $\alpha$ parameters; (ii) relevance to the construct the item was intended to measure; and (iii) contribution to the shape of the information function for the particular subscale.

Confirmatory Factor Analysis. A confirmatory factor analysis (CFA) was conducted with AMOS $21^{21}$ to test the fit of the short form to the original factor structure to substantiate construct validity. Using the retained items from the IRT analysis, the hypothesized model was fit to the data using maximum likelihood estimation. Model data fit was analyzed using the comparative fit index $(\mathrm{CFI}),{ }^{22}$ the root-mean-square error of approximation (RMSEA), ${ }^{23}$ and the standard root-meansquare residual (SRMR). The literature suggests CFI $>0.95$, RMSEA $<0.06$, and SRMR $<0.08$ for good fit and CFI $>0.90$, RMSEA $<0.10$, and SRMR $<0.10$ for acceptable fit. ${ }^{22,24,25}$

Phase 2: Cross-Validation Sample. Once the ALSSQOLSF was created, a prospective study of ALS patients attending 10 multidisciplinary clinics was conducted to test its validity. Inclusion and exclusion criteria were identical to those in Phase 1. Study measures were completed on site via interviews with patients by ALS clinic personnel who were part of the site study team. Patient enrollment and data collection were performed from January through December 2014.

Participants' demographics and descriptive information were recorded, including the ALS Functional Rating ScaleRevised (ALSFRS-R) ${ }^{26}$ After completion of the ALSSQOL-SF, participants completed the following measures to evaluate the construct, convergent, and discriminant (divergent) validity of the overall ALSSQOL-SF and its subscales. These measures were selected to replicate previous methodology used for the development of the ALSSQOL-R, and were determined to be reliable and valid ${ }^{11,27-29}$ : the McGill Quality of Life Single-Item Score (MQOL-SIS), ${ }^{30}$ modified to assess QOL over the past 7 days, rather than 2 days, appears on the ALSSQOL-SF as the first question, although scored separately. This measure was used for testing of validity of the ALSSQOL-SF's assessment of global QOL. The World Health Organization Quality of Life Instrument-Brief version (WHOQOL-BREF) ${ }^{31}$ domain scores (Psychological, Social Relationships, Environment, Physical Health) were compared with the ALSSQOL-SF Average Total Score or to subscales as follows: Psychological domain was compared with the Negative Emotion subscales; Social Relationships domain was compared with the Interaction with People and the Environment and Intimacy subscales (no specific comparison measure of intimacy was used, because most measures require information from both partners); Environment domain was compared with the Interaction with People and the Environment subscale; and Physical Health domain was compared with the Physical Symptoms subscale. The Idler Index of Religiosity $^{32}$ (IIR), used to evaluate the ALSSQOL-SF Religiosity subscale, has 2 subscales-Public Religiousness and Private Religiousness. The Satisfaction with Life Scale ${ }^{33}$ (SWLS) was included to evaluate the ALSSQOL-SF Interaction with People and Their Environment subscale. The Center for Epidemiological Studies-Depressed Mood Scale ${ }^{34}$ (CES-D) was compared with the Negative Emotion subscale. The 18-item Brief Symptom Inventory ${ }^{35}$ (BSI) was also compared with the Negative Emotion subscale.

Statistical Analyses. Descriptive statistics were conducted using mean and frequency data. Some demographic data were not completed by the participants, and were de-identified before they could be retrieved, resulting in minor variation in 
sample size for demographic data. The primary analysis of the study used CFA to verify the structure and content of the ALSSQOL-SF. The relationships between subscales of the ALSSQOL-SF and external measures of the same constructs were evaluated using Pearson's product-moment correlations. Hierarchical linear regression was used to evaluate the subscales' ability to predict global QOL. All analyses were conducted with SPSS (version 22.0) ${ }^{36}$ and AMOS. ${ }^{21} P<0.05$ was considered significant. A minimal sample size of 200 was determined to be sufficient to conduct IRT, and greater than needed to achieve 0.80 power with a medium effect size and $\alpha=0.01$ for all remaining analyses. Two hundred subjects is considered a small but reasonable sample for IRT $^{37}$; a larger sample would have been more difficult to obtain from this population within the planned time-frame of the study. The most important factors in determining the appropriate sample size for this analysis are dependent on how much information could be extracted from the data, and for the standard error for the estimated parameters for the information functions to show that they had similar shapes. This was accomplished here.

\section{RESULTS}

Phase 1: Creation of the ALSSQOL-SF. There were 389 participants (59\% men). Ages ranged from 27 to 88 (mean 61, SD 11) years. Duration of symptoms at study enrollment averaged 39.7 months (SD 39.2 months, median 26 months, range 3-232 months; $N=324$ ). Length of time from diagnosis to study enrollment averaged 21.5 months (SD 27.0 months, median 12.5 months, range 0-223 months; $N=362$ ). ALSFRS-R scores $(N=385)$ ranged from 6 to 48 (mean 33.11, SD 8.0).

Table S1 (refer to Supplementary Material online) presents the estimated $\alpha$ and $\beta$ parameters for the ALSSQOL-R. Figure 1 presents the final information functions for each subscale. The findings across the 6 graphs indicate that the ALSSQOL-SF had slightly greater imprecision compared with the ALSSQOL-R, although this is to be expected given that the ALSSQOL-SF has fewer items. Table 1 provides a structural comparison between the original and short form. Twenty items were retained, reducing the length of the ALSSQOL-R by $>50 \%$. The Physical Symptom subscale was altered the least, with only 1 item removed, given that any further reductions to the original scale would have reduced reliability to below acceptable levels. Negative Emotion and Interaction with People and the Environment subscales were reduced the most.

For the CFA, the hypothesized model is displayed in Figure 2, using the retained items from the IRT analysis. The CFA results show the model fit to be acceptable: $\chi^{2}{ }_{(164)}=527.20 ; P<0.001 ; \mathrm{CFI}=0.876$; RMSEA $=0.076$; and $\mathrm{SRMR}=0.078$. Table 2 shows the factor loadings by item for the ALSSQOL-SF. All items loaded onto their respective factors as suggested by the model, supporting the same factor structure as the original ALSSQOL-R. Correlations between factors (Table 3) were mostly low, ranging from 0.06 to 0.42 , and were similar to results for the ALSSQOL-R. ${ }^{13}$ Table 3 presents the intercorrelations among factors on the ALSSQOL-SF. It shows that the results of the original principal component analysis with orthogonal varimax rotation that was conducted to reduce the ALSSQOL to the ALSSQOL-R remained intact when the ALSSQOL-R was further reduced to the ALSSQOL-SF. Thus, the individuality of the factors remained intact, and the correlations between subscales are generally low to not significant.

A copy of the current version of the ALSSQOL-SF is presented in the Supplementary Material (online).

Phase 2: Cross-Validation Sample. There were 162 participants. The majority were male (59.3\%; $N=96$ ), with a mean age of 60.5 years (SD 10.99 years, range 26-85 years; $N=154$ ). The mean ALSFRS-R score was 31.08 (SD 9.15, range 1-48, median 32). Time from symptom onset to data collection averaged 35.6 months (SD 34 months, median 27.5 months, range 4-273 months), and duration of time from diagnosis to study entry averaged 20.8 months (SD 30.2 months, median 13 months, range 0-253 months). Completion of the ALSSQOL-SF required 2-4 minutes. One hundred fifty-four patients completed all items on the ALSSQOL-SF, and were included in the CFA without imputation. Series-mean imputation was used for missing items to allow more complete data for additional analyses.

\section{Evaluation of the ALSSQOL-SF. The initial CFA} run estimated a negative error variance for item 12 and, as a result, the model was unable to converge. A negative error variance may have been caused by the small sample size, ${ }^{38}$ or a Heywood case $^{39}$ as item 12 is an indicator variable for the Religiosity factor, which is only comprised of 2 items. ${ }^{40} \mathrm{~A}$ Heywood case means the factor is possibly not contributing unique variance. However, the negative error variance was not statistically different from $0(P>0.05)$. Thus, the negative value is most likely due to sampling error as a deficiency in the model and can safely be constrained to a non-negative value to allow the model to converge. After constraining the error variance for item 12 to 0 , the model converged with a similar fit to the previous CFA: $\chi_{(165)}^{2}=349.92 ; \quad P<0.001 ; \quad \mathrm{CFI}=0.857 ; \quad$ RMSEA $=$ 0.083 ; and SRMR $=0.10$.

ALSSQOL-SF Scores. The mean score of all ALSSQOL-SF items (Average Total Score) was 6.5 (SD 1.1, range 3.7-9.4). Scores for subscales are provided in Table 4. Range of scores showed maximum use of the scale (minimum 0, maximum 10) for most of the subscales. 

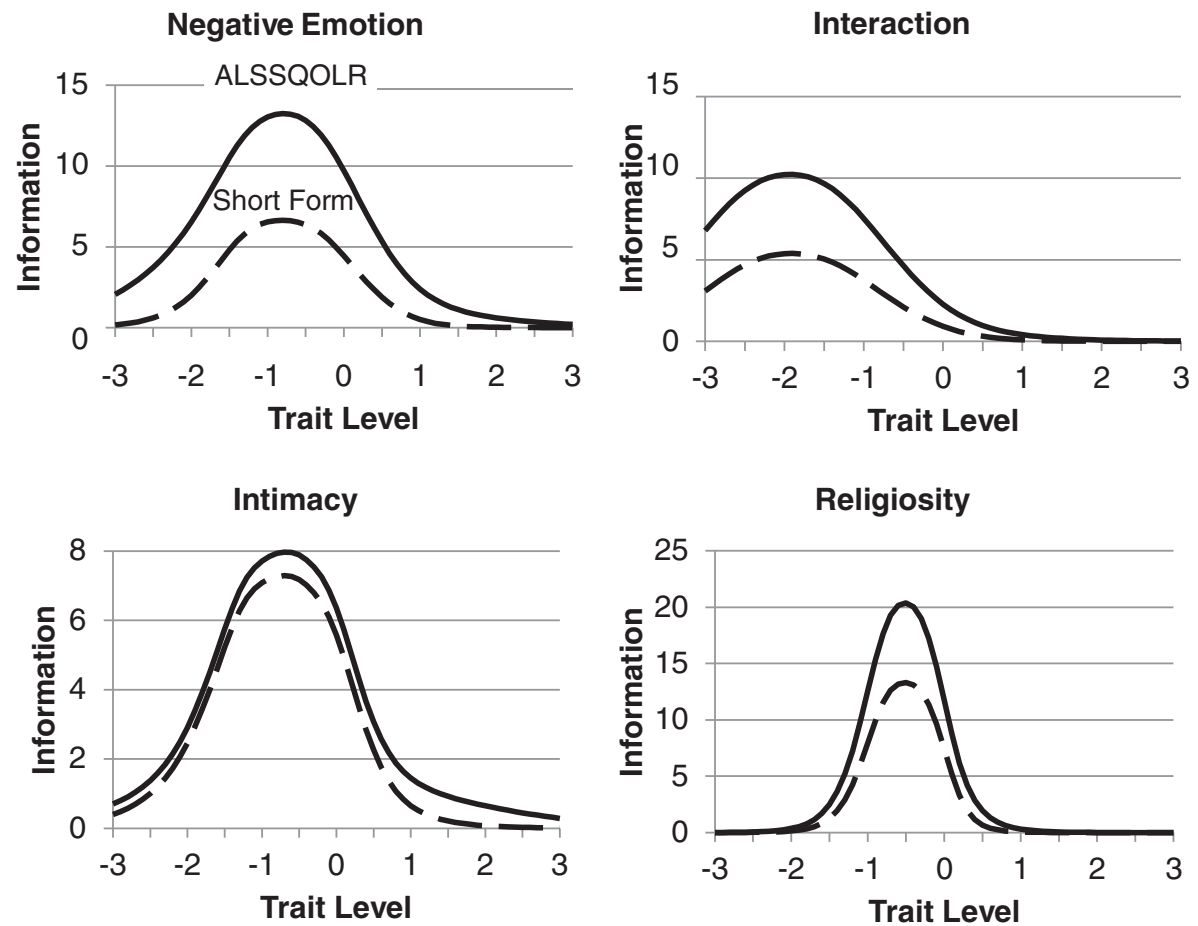

Physical Symptoms
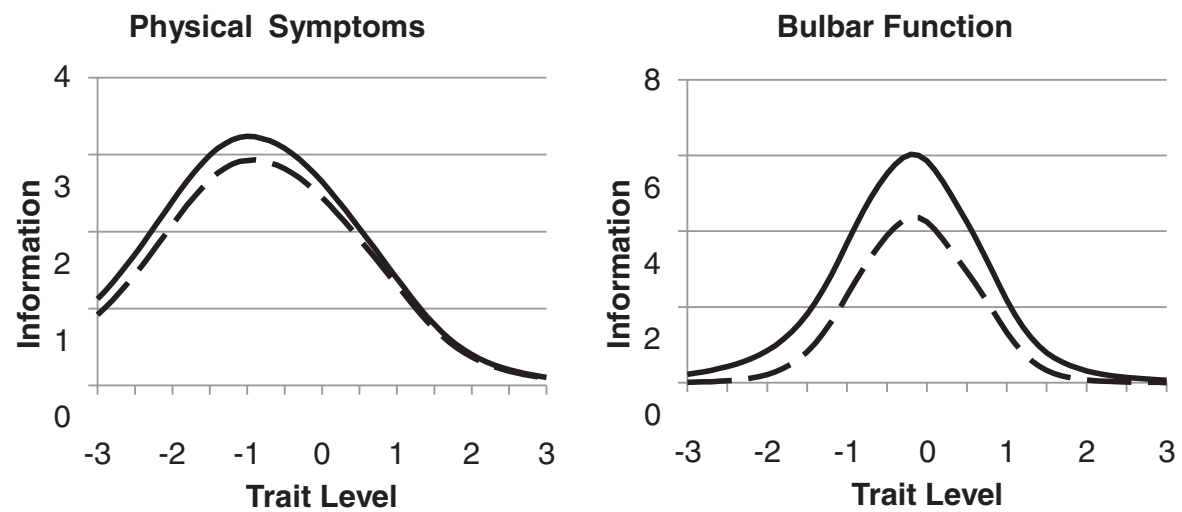

FIGURE 1. Information functions by subscale for the ALS-Specific Quality of Life Instrument-Revised (ALSSQOL-R) and its short form, the ALSSQOL-SF. In an Item Response Theory (IRT) context, information is the corollary to reliability and can be expressed as the reciprocal of the standard error conditional for a particular trait level: $I(\theta)=1 / \mathrm{SE}(\theta)^{2}$. Thus, more information indicates more precision in measurement. In addition, trait level refers to an individual's capacity (score) for the particular construct measured by the subscale, with 0 representing the average and higher numbers reflecting more characteristics relative to the data in the sample. The ALSSQOL-SF has slightly greater imprecision compared to the ALSSQOL-R, but the information functions display a consistent pattern between both forms, maximizing accuracy at the same points along the trait scale. Interaction $=$ Interaction with People and the Environment.

Table 1. Structural comparison of ALS-Specific Quality of Life Instrument-Revised and Short Form

\begin{tabular}{lcccc} 
Scale & ALSSQOL-R & ALSSQOL-SF & ALSSQOL-R reliability & ALSSQOL-SF reliability \\
\hline Negative Emotion & 13 items (28\%) & 3 items (15\%) & 0.91 & 0.86 \\
Interaction & 11 items (24\%) & 4 items (20\%) & 0.87 & 0.80 \\
Intimacy & 7 items (15\%) & 4 items (20\%) & 0.81 & 0.82 \\
Religiosity & 4 items (9\%) & 2 items (10\%) & 0.92 & 0.89 \\
Physical Symptoms & 6 items (13\%) & 5 items (25\%) & 0.71 & 0.70 \\
Bulbar Function & 5 items (11\%) & 2 items (10\%) & 0.83 & 0.81 \\
\hline
\end{tabular}

Reliability was measured using Cronbach's $\alpha$. ALSSQOL-R, ALS-Specific Quality of Life Instrument-Revised; ALSSQOL-SF, ALS-Specific Quality of Life Instrument-Short Form; Interaction, Interaction with People and the Environment. 


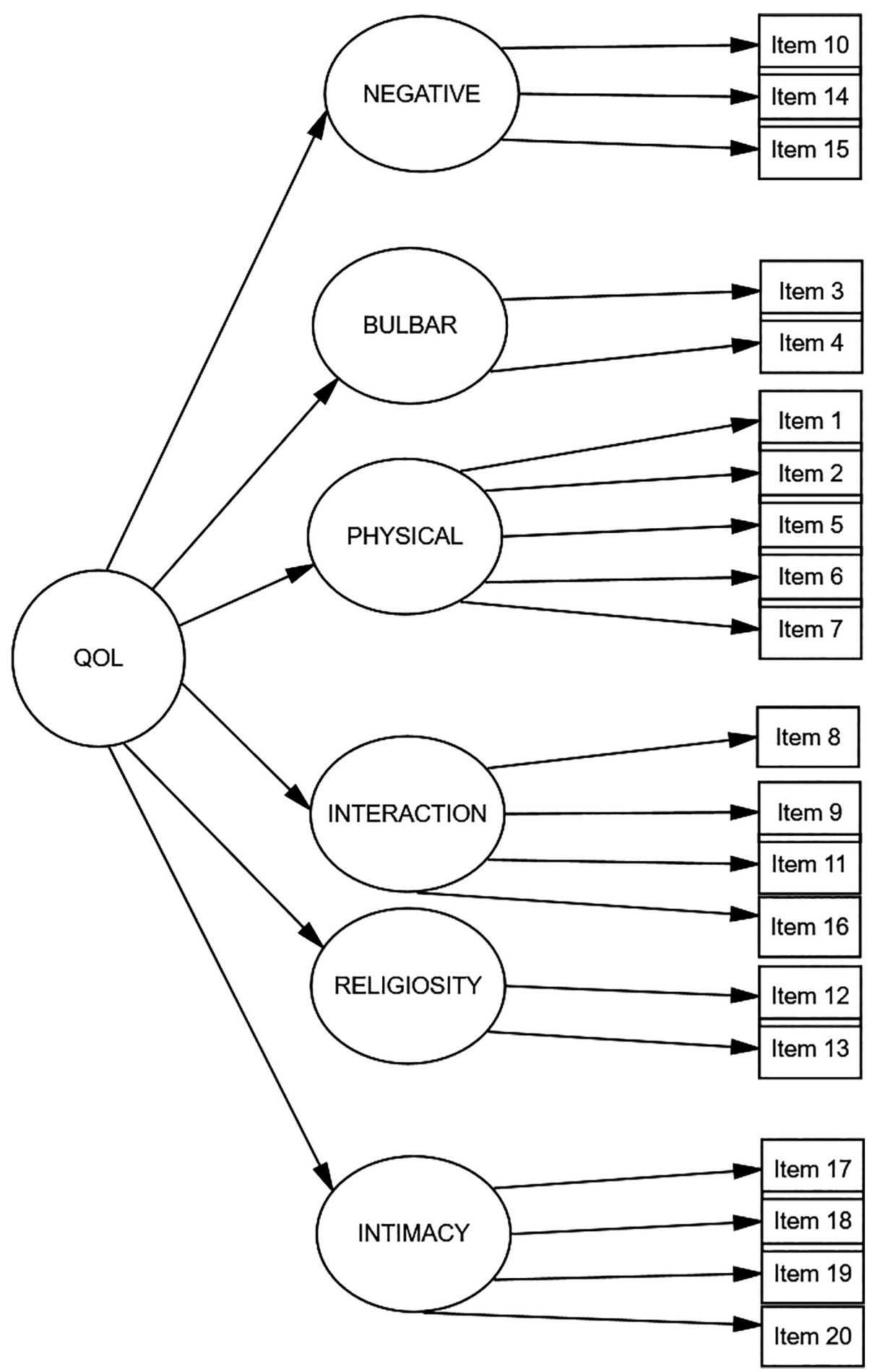

FIGURE 2. Hypothesized second-order model of factorial structure for the ALS-Specific Quality of Life Instrument-Short Form (ALSSQOLSF). The items contributing to each of the 6 subscales of the ALSSQOL-SF are shown. Item numbers correspond to the specific items of the ALSSQOL-SF (see Supplementary Material online for the complete questionnaire). QOL = ALSSQOL-SF; Negative = Negative Emotion; Bulbar = Bulbar Function; Physical $=$ Physical Symptoms; Interaction $=$ Interaction with People and the Environment.

Validity Testing. Correlations are reported in Table 5 for the ALSSQOL-SF Average Total and Subscale Scores as assessments of convergent, divergent, and construct validity. The ALSSQOL-SF Average Total Score and ALSFRS-R showed a significant, moderate correlation. Moderate, positive, significant correlations were found between the ALSSQOL-SF Average Total Score and other measures of global QOL, including MQOL-SIS and WHOQOL subscales and SWLS. Hierarchical multiple regression conducted using MQOL-SIS as the dependent variable, and the subscales of the ALSSQOL-SF as the 
Table 2. Confirmatory factor analysis: standardized loadings for the ALS Specific Quality of Life Instrument-Short Form

\begin{tabular}{|c|c|c|c|c|c|c|}
\hline Short Form items & NE & IPE & IN & $\mathrm{RE}$ & PS & $\mathrm{BF}$ \\
\hline 10. I have been depressed & 0.868 & & & & & \\
\hline 14. I have felt hopeless & 0.737 & & & & & \\
\hline 15. I have felt sad & 0.838 & & & & & \\
\hline 8. The world has been caring and responsive to my needs & & 0.737 & & & & \\
\hline 9. I have felt supported & & 0.840 & & & & \\
\hline 11. Relationships have been satisfying & & 0.629 & & & & \\
\hline 16. I have enjoyed the beauty of my surroundings & & 0.592 & & & & \\
\hline 17. My desire for emotional intimacy has been strong & & & 0.787 & & & \\
\hline 18. I have shared emotional intimacy with others & & & 0.762 & & & \\
\hline 19. My desire for physical intimacy has been strong & & & 0.702 & & & \\
\hline 20. I have shared physical intimacy with others & & & 0.674 & & & \\
\hline 12. My religion has been a source of strength/comfort to me & & & & 0.969 & & \\
\hline 13. I consider myself to have been religious or spiritual & & & & 0.834 & & \\
\hline 1. Pain & & & & & 0.468 & \\
\hline 2. Fatigue & & & & & 0.676 & \\
\hline 5. My Strength and Ability to Move & & & & & 0.505 & \\
\hline 6. Sleep & & & & & 0.528 & \\
\hline 7. I have felt physically terrible & & & & & 0.649 & \\
\hline 3. Excessive saliva & & & & & & 0.946 \\
\hline 4. Speaking & & & & & & 0.724 \\
\hline
\end{tabular}

NE, Negative Emotion; IPE, Interaction with People and the Environment; IN, Intimacy; RE, Religiosity; PS, Physical Symptoms; BF, Bulbar Function.

Table 3. Correlations between factors on the ALS-Specific Quality of Life Instrument-Short Form

\begin{tabular}{lccccc}
\hline & & & & & \\
& IPE & IN & RE & PS & BF \\
\hline Negative Emotion & $0.33^{\dagger}$ & $0.17^{\dagger}$ & $0.11^{*}$ & $0.42^{\dagger}$ & 0.06 \\
IPE & & $0.30^{\dagger}$ & $0.30^{\dagger}$ & $0.22^{\dagger}$ & 0.07 \\
Intimacy & & & $0.25^{\dagger}$ & 0.06 & 0.08 \\
Religiosity & & & & 0.06 & -0.07 \\
Physical Symptoms & & & & & $0.11^{*}$ \\
\hline
\end{tabular}

NE, Negative Emotion; IPE, Interaction with People and the Environment; IN, Intimacy; RE, Religiosity; PS, Physical Symptoms; BF, Bulbar Function. $* P<0.05$.

${ }^{\dagger} P<0.001$

Table 4. Normative/Mean scores of quality of life in total patient sample completing the ALS Specific Quality of Life InstrumentShort Form

\begin{tabular}{lcc}
\hline & & \\
Measure & Mean $(S D)$ & Range \\
\hline MQOL-SIS $(N=162)$ & $7.0(2.1)$ & $0-10$ \\
ALSSQOL-SF Average Total Score $(N=160)$ & $6.5(1.1)$ & $3.7-9.3$ \\
ALSSQOL-SF NE $(N=161)$ & $5.3(1.2)$ & $1.0-7.7$ \\
ALSSQOL-SF IPE $(N=161)$ & $8.6(1.4)$ & $3.0-10.0$ \\
ALSSQOL-SF IN $(N=159)$ & $6.7(2.3)$ & $0.0-10.0$ \\
ALSSQOL-SF RE $(N=162)$ & $6.7(3.5)$ & $0.0-10.0$ \\
ALSSQOL-SF PS $(N=162)$ & $5.3(2.3)$ & $0.0-10.0$ \\
ALSSQOL-SF BF $(N=162)$ & $6.4(3.2)$ & $0.0-10.0$ \\
ALSSQOL-SF Total Score $(N=160)$ & $130.1(21.9)$ & $74-187$ \\
\hline
\end{tabular}

MQOL-SIS, McGill Quality of Life Single Item Scale; ALSSQOL-SF, ALS-Specific Quality of Life Instrument-Short Form; NE, Negative Emotion; IPE, Interaction with People and the Environment; IN, Intimacy; RE, Religiosity; PS, Physical Symptoms; BF, Bulbar Function.

independent variable revealed only 3 (Negative Emotion, Interaction with People and the Environment, and Physical Symptoms) of the 6 variables were significant predictors of MQOL-SIS, accounting for
$2.7 \%, \quad 9.6 \%$, and $7.8 \%$, respectively $[F$ $(3,153)=5.083, P=0.026]$, and a total of $44.7 \%$ of the variability. The $\beta$ weights are presented in Table S2 (online).

Correlations of the Negative Emotion subscale with the Depression and Anxiety subscales of the 18-item BSI, CESD, and WHOQOL Psychological domain, were moderate to small. Correlations between Interaction with People and the Environment and the WHOQOL Social Relationships subscale, WHOQOL Environment subscale, and SWLS were significant, and moderate to small. No direct measure of intimacy was used. However, the ALSSQOL-SF Intimacy subscale and WHOQOL Social Relationships correlated moderately. Strong, positive, and significant relationships were found between the Religiosity subscale and both the Public Religiosity subscale of the IIR and the total/overall IIR score.

When assessing correlations of the Physical Symptoms subscale, a positive, moderate correlation was found with the total ALSFRS-R score. A closer comparison of subscales of the ALSFRS-R shows significant correlations among all, but most closely with ALSFRSR Fine Motor and ALSFRS-R Gross Motor Functioning, and less so with ALSFRS-R Speech and Respiratory. The relationship between the ALSSQOL-SF Physical Symptoms and the WHOQOL Physical domain was strong and positive. Bulbar Function demonstrated strong, positive, and significant correlations with the ALSFRS-R Speech subscale. The relationship between this subscale and the ALSFRS-R Respiratory subscale was significant, but weak. There was not a significant correlation between the ALSSQOL-SF Bulbar Function and the WHOQOL Physical domain. 
Table 5. Correlations between ALS-Specific Quality of Life Instrument-Short Form subscales and measures to test convergent, divergent, and construct validity ( $N=162$ unless otherwise noted)

\begin{tabular}{|c|c|c|c|c|c|c|c|}
\hline Measures & ATS & NE & IPE & PS & IN & RE & $\mathrm{BF}$ \\
\hline ALSFRS-R Total & $0.40^{\dagger}$ & & & $0.48^{\dagger}$ & & & $0.29^{\dagger}$ \\
\hline Speech & & & & $0.17 *$ & & & $0.79^{\dagger}$ \\
\hline Fine Motor & & & & $0.37^{\dagger}$ & & & 0.06 \\
\hline Gross Motor & & & & $0.44^{\dagger}$ & & & -0.04 \\
\hline Respiratory & & & & $0.34^{\dagger}$ & & & $0.23^{\dagger}$ \\
\hline MQOLSIS & $0.34^{\dagger}$ & $0.28^{\dagger}(N=161)$ & $0.28^{\dagger}(N=161)$ & $0.25^{\dagger}$ & $0.10(N=159)$ & 0.09 & 0.11 \\
\hline WHOQOL Physical & $0.47^{\dagger}(N=161)$ & & & $0.60^{\dagger}(N=161)$ & & & $0.118(N=161)$ \\
\hline WHOQOL Psychological & $0.52^{\dagger}(N=158)$ & $-0.37^{\dagger}(N=157$ & & & $0.27 *(N=155)$ & & \\
\hline WHOQOL Social & $0.57^{\dagger}(\mathrm{N}=160)$ & & $0.49^{\dagger}(N=159)$ & & $0.42^{\dagger}(N=157)$ & & \\
\hline WHOQOL Environment & $0.39^{+}(N=159)$ & & $0.37^{\dagger}(N=158)$ & & & & \\
\hline SWLS & $0.44^{\dagger}(N=161)$ & $0.33^{\dagger}(N=160)$ & $0.37^{\dagger}(N=160)$ & $0.21 *(N=161)$ & $0.28^{\dagger}(N=158)$ & $\begin{array}{l}0.16 * \\
(N=161)\end{array}$ & $0.10(N=161)$ \\
\hline CESD & & $-0.38^{\dagger}(N=160)$ & & & & & \\
\hline BSI-18 Depression & & $-0.37^{\dagger}(N=159)$ & & & & & \\
\hline BSI-18 Anxiety & & $-0.29^{\dagger}(N=159)$ & & & & & \\
\hline BSI-18 Somatization & & $-0.13(N=159)$ & & & & & \\
\hline BSI-18 Total & & $-0.33^{\dagger}(N=159)$ & & & & & \\
\hline IIR Public & & & & & & $0.63^{\dagger}(N=151)$ & \\
\hline IIR Private & & & & & & $-0.21^{\dagger}(N=161)$ & \\
\hline IIR Total & & & & & & $0.57^{\dagger}(N=151)$ & \\
\hline
\end{tabular}

ATS, ALSSQOL-SF Average Total Score; NE, Negative Emotion; IPE, Interaction with People and the Environment; IN, Intimacy; RE, Religiosity; PS, Physical Symptoms; BF, Bulbar Function; MQOLSIS, McGill Quality of Life Single Item Scale; WHOQOL, World Health Organization Quality of Life; SWLS, Satisfaction with Life Scale; CESD, Center for Epidemiological Studies Depressed Mood Scale; BSI-18, 18-item Brief Symptom Inventory; IIR, Idler Index of Religiosity.

$* P<0.05$.

${ }^{\dagger} P<0.001$

\section{DISCUSSION}

The ALSSQOL-R was successfully modified to a briefer version, the ALSSQOL-SF, while maintaining the goal of being a valid, disease-specific QOL instrument. The ALSSQOL-SF retains the value of reflecting areas that patients volunteered as most important to their QOL, thus potentially assisting health-care professionals with intervention planning and comprehensive interprofessional care. The new short form contains less than half of the number of items of previous versions, which shortened time to completion from 15-25 minutes to 2-4 minutes.

The results of Phase 1 represent a vast improvement of the model fit compared with the original ALSSQOL-R. ${ }^{13}$ The improvement in fit may be due to removal of items that measured different facets of the constructs and/or partially measured other characteristics, decreasing the efficiency of the scale. This improvement resulted in information being maximized in similar regions as in the original form, contributing to optimal precision with fewer items.

We chose to retain a $0-10$ rating scale on the ALSSQOL-SF for administration/scoring of the measure because it allows for more sensitivity to subtle changes in clinical symptoms for individuals in a treatment setting. It is also consistent with other scales to which patients are accustomed, such as pain scales. The IRT scoring was used for validation of the measure, but is not implemented in regular use of the measure. It is likely, however, that the correlation between participant scores on both scales would be close to a perfect correlation. Thus, although there may be less precision in someone's category score, it leads to the same inference and therefore maintains score validity. Future studies could evaluate a reduction in scale from $0-10$ to $0-5$ for clinical and research utility.

The Negative Emotion and Interaction with People and the Environment subscales originally had the most items compared with the other subscales. They also had the greatest reduction in items, and therefore, clinically, these domains lost the most information. However, given that these were 2 of the more stable subscales of the ALSSQOL-R, the shortened versions are sufficient for representing and evaluating these constructs.

Phase 2 demonstrated that the ALSSQOL-SF and its subscales have construct, convergent, and divergent (discriminant) validity. The weak to moderate correlations between the ALSQOL-SF Average Total Score and general global measures of QOL suggest that they measure related but not overlapping constructs.

The significant, yet small to moderate correlations between the Negative Emotion subscale and the 18-item BSI and CESD suggest that negative emotion is more than just anxiety or depression for persons with ALS, as previously identified. ${ }^{12}$ The Negative Emotion subscale appears to be appropriate as a brief screening tool, but additional evaluation of 
depressed mood, anxiety, coping, and adjustment should be performed if scores on this subscale are low.

The Interaction with People and the Environment subscale represents social relationships and existential considerations such as one's appraisal of and reciprocal relationship with the environment. The moderate to small correlations between this subscale and the WHOQOL Social Relationships, WHOQOL Environment, and SWLS verify the complexity of these concepts, shedding additional light on topics of importance to ALS patients.

The concept of intimacy is not directly assessed in global or general QOL measures. Measuring intimacy in ALS patients and monitoring its change over time may be an important route of intervention and for problem-solving ways to bolster QOL in ALS patients, as it can be achieved despite physical dysfunction and decline.

The Religiosity subscale suggests that engaging in religious practices as part of a community may have intrinsic and social benefits that contribute to overall QOL. However, this does not mean that more is better. Rather, the Religiosity subscale may allow for a more comprehensive needs assessment and may give insight as to whether referrals for religious support are warranted if there is a concordant decline in Religiosity and in the overall QOL or the Negative Emotion subscale.

Concurrent validity of the ALSSQOL-SF Physical Function subscale is supported by the strong, positive, and significant relationship between that scale and the WHOQOL Physical domain, both of which evaluate patients' perceptions of physical functioning. The convergent and construct validity of the ALSSQOL-SF Bulbar Function subscale was evident via its correlations with the ALSFRS-R Speech subscale, whereas the lack of correlations with the ALSFRS-R Fine Motor and Gross Motor subscales and the WHOQOL Physical domain demonstrate appropriate divergent/discriminant validity. This supports Bulbar Function and Physical Symptoms remaining as 2 distinct subscales of the ALSSQOLSF. Earlier research has also supported this distinction, as verbal communication was found to be closely related to QOL, ${ }^{41}$ whereas overall physical function has often been shown to not correlate with global QOL.

Comparison of ALSSQOL-SF to the ALSSQOL-R. In evaluating the ALSSQOL-SF's ability to predict variance in the global QOL measure, MQOL-SIS, the hierarchical multiple regressions for each of the 2 ALSSQOL measures were similar but not the same. Only 2 of the 3 predictors (Negative Emotion and Physical Symptoms) were shared between the 2 instruments, and ALSSQOL-R's more expansive
Negative Emotion subscale predicted $37 \%$ of the global QOL in the study sample compared with $2.7 \%$ of the variance being accounted for by the Negative Emotion subscale of the ALSSQOL-SF. Comparisons of the 2 versions of the ALSSQOL further validate the factor structure, and reflect the differences in the information provided by each. Comparisons of the 2 instruments also reveal that the new measure has weaker correlations on subscales with external measures, ${ }^{13}$ which is not surprising, given the reduction in items. This suggests that the short form subscales may be less reliable standalone measures of these constructs than the ALSSQOL-R subscales. The 2 versions of the measure provide flexibility. For instance, clinicians may choose to use the ALSSQOL-R for the initial evaluation of patients, and conduct follow-up assessments with the short form. Another option is to routinely use the short form for screening and follow-ups, and to administer the ALSSQOL-R subscales only when concern is raised in a specific area. Researchers may prefer the ALSSQOL-R, given its provision of more information and sensitivity to subtle changes in broadly assessed domains. The flexibility in choosing the version is supported by the similar overall QOL score obtained using the ALSSQOL-SF (6.5), ALSSQOL-R (6.8), and ALSSQOL (7.1). ${ }^{11,13}$

There were methodological limitations to this study. Patient samples were drawn from individuals attending large ALS referral centers, and thus may not be representative of the ALS patient population as a whole, despite a similar age and gender distribution to that of the general ALS population. ${ }^{42,43}$ Data from the 2 studies were collected at different time periods, and it is possible that patient care or values relating to QOL changed in the interval. The close match of results from the ALSSQOL-R and ALSSQOL-SF studies diminishes this concern. Our studies collected data through interviews; clinics adopting the ALSSQOL-SF may prefer to use computer-based or hard-copy self-completion of the instrument. Although our previous research showed no difference in results based on administration method, ${ }^{44}$ it is possible that clinics may find different results using different administration methods.

Limitations also exist regarding measures selected for assessment of validity. A more recent version of the CESD was developed and released in 2004, but few appropriate normal scores were available at the time the current study was being developed. The IIR only has 4 items, and therefore less variability, despite evidence supporting its reliability and validity. $^{32}$ Intimacy measures have been validated on younger, healthy populations, ${ }^{45,46}$ or require comparison of dyad responses, which was not practical for this research. Last, the items on the ALSSQOL, and therefore the ALSSQOL-R and -SF, are 
distributed among several of the subscales, and therefore the subscales are defined and compared by the dominant concepts reflected; no existential comparative measure was used.

In conclusion, for purposes of measuring QOL as a holistic construct and understanding factors that affect patients' reports of QOL, the ALSSQOL-SF appears to be an efficient, quickly administered instrument with good psychometric properties. Clinicians and researchers should consider the advantages and limitations of the ALSSQOL-R vs. the ALSSQOL-SF when choosing one or the other for a specific use. Guidelines for scoring and routine clinical use of the ALSSQOL-SF will appear in a forthcoming manual.

Ethical publication statement: We (the authors) confirm that we have read the Journal's position on issues involved in ethical publication and affirm that this report is consistent with those guidelines.

\section{REFERENCES}

1. Burns TM, Graham CD, Rose MR, Simmons Z. Quality of life and measures of quality of life in patients with neuromuscular disorders. Muscle Nerve 2012;46:9-25.

2. Simmons Z. Patient-perceived outcomes and quality of life in ALS. Neurotherapeutics 2014;12:394-402.

3. World Health Organization, Division of Mental Health and Prevention of Substance Abuse. WHOQOL: measuring quality of life. 1997. Available at http://www.who.int/mental_health/media/68.pdf. Accessed June 17, 2016.

4. Neudert C, Wasner M, Borasio GD. Individual quality of life is not correlated with health related quality of life or physical function in patients with amyotrophic lateral sclerosis. J Palliat Med 2004;7:551-557.

5. Simmons Z, Bremmer BA, Robbins RA, Walsh SM, Fischer S. Quality of life in ALS depends on factors other than strength and physical function. Neurology 2000;55:388-392.

6. Felgoise SH, Stewart JL, Bremer BA, Walsh SM, Bromberg MB, Simmons Z. The SEIQoL-DW for assessing quality of life in ALS: strengths and limitations. Amyotroph Lateral Scler 2009;10: 456-462.

7. Robbins RA, Simmons Z, Bremer BA, Walsh SM, Fischer S. Quality of life in ALS is maintained as physical function declines. Neurology 2001; 56:442-444.

8. Neudert C, Wasner M, Borasio GD. Patients' assessment of quality of life instruments: a randomized study of SIP, SF-36 and SEIQOL-DW in patients with amyotrophic lateral sclerosis. J Neurol Sci 2001;191:103-109.

9. Bromberg MB, Forshew DA. Comparison of instruments addressing quality of life in patients with ALS and their caregivers. Neurology 2002;58:320-322.

10. Goldstein LH, Atkins L, Leigh PN. Correlates of quality of life in people with motor neuron disease (MND). Amyotroph Lateral Scler Other Motor Neuron Disord 2002;3:123-129.

11. Simmons Z, Felgoise SH, Bremer BA, Walsh SM, Hufford DJ, Bromberg MB, et al. The ALSSQOL: balancing physical and non-physical factors in assessing quality of life in ALS. Neurology 2006; 67:1659-1664

12. Simmons Z, Felgoise SH, Rodriguez JL, Walsh SM, Bremer BA, Stephens HE. Validation of a shorter ALS-specific quality of life instrument: the ALSSQOL-R. Neurology 2010;74(suppl 2):A177-A178.

13. Felgoise SH, Walsh SM, Stephens HE, Brothers A, Simmons Z. The ALS Specific Quality of Life-Revised (ALSSQOL-R) user's guide, version 1.0, version date 6/14/2011. Available at http://www.pennstatehershey. org/c/document_library/get_file?uuid= b9de0a6a-9c1d-4f77-bdf0-5c6c846e018e\&groupId=22147. Accessed February 2, 2018.

14. Stephens H, Young J, Felgoise S, Simmons Z. A qualitative study of multidisciplinary ALS clinic use in the United States. Amyotroph Lateral Scler Frontotemporal Degener 2016;17:55-61.

15. Brooks BR, Miller RG, Swash M, Munsat TL, for the World Federation of Neurology Research Group on Motor Neuron Diseases. Amyotroph Lateral Scler Other Motor Neuron Disord 2000;1:293-299.

16. Hambleton RK, Swaminathan H, Rogers HJ. Fundamentals of item response theory. Newbury Park, CA: Sage; 1991.
17. Lord FM. Practical applications of item characteristic curve theory. J Educ Meas 1977;14:117-138.

18. Muraki E. Fitting a polytomous item response model to Likert-type data. App Psychol Meas 1990;14:59-71.

19. Muraki E, Bock D. PARSCALE for Windows (version 4.1). Chicago: Scientific Software International; 2003.

20. Embretson SE, Reise SP. Item response theory for psychologists. Mahwah, NJ: Lawrence Erlbaum; 2000.

21. Arbuckle JL. AMOS (version 21.0) [computer program]. Chicago: IBM SPSS; 2012.

22. Bentler P. Comparative fit indexes in structural models. Psychol Bull 1990;107:238-246.

23. Steiger JH. Structural model evaluation and modification: an interval estimation approach. Multivariate Behav Res 1990;25:173-180.

24. Browne M, Cudeck R. Alternative ways of assessing model fit. In: Bollen K, Long J, editors. Testing structural equation models. Newbury Park, CA: Sage; 1993. p 136-162.

25. $\mathrm{Hu} \mathrm{L}$, Bentler P. Cutoff criteria for fit indexes in covariance structure analysis: Conventional criteria versus new alternatives. Struct Equ Model 1999;1:1-55.

26. Cedarbaum JM, Stambler N, Malta E, Fuller C, Hilt D, Thurmond B, et al. The ALSFRS-R: a revised ALS functional rating scale that incorporates assessments of respiratory function. J Neurol Sci 1999;169:13-21.

27. Felgoise SH, Chakraborty $\mathrm{BH}$, Bond E, Rodriguez J, Bremer BA, Walsh SM, et al. Psychological morbidity in ALS: the importance of psychological assessment beyond depression alone. Amyotroph Lateral Scler 2010;11:351-358.

28. Bremer BA, Simone AL, Walsh S, Simmons Z, Felgoise SH. Factors supporting quality of life over time for individuals with amyotrophic lateral sclerosis: the role of positive self-perception and religiosity. Ann Behav Med 2004;28:119-125.

29. Walsh S, Bremer BA, Felgoise SH, Simmons Z. Religiousness is related to quality of life in patients with ALS. Neurology 2003;60:1527-1529.

30. Cohen SR, Mount BM, Strobel MG, Bui F. The McGill Quality of Life Questionnaire: a measure of quality of life appropriate for people with advanced disease. A preliminary study of validity and acceptability. Palliat Med 1995;9:207-219.

31. WHOQOL Group. The World Health Organization Quality of Life Assessment (WHOQOL): development and general psychometric properties. Soc Sci Med 1998;46:1569-1585.

32. Fetzer Institute, National Institute on Aging Working Group. Multidi mensional measurement of religiousness/spirituality for use in health research. Kalamazoo, MI: Fetzer Institute; 2003.

33. Diener E, Emmons RA, Larsen RJ, Griffin S. The Satisfaction with Life Scale. J Person Assess 1985;49:71-75.

34. Radloff LS. The CES-D Scale: a self-report depression scale for research in the general population. Appl Psych Meas 1977;1:385-401.

35. Derogatis LR. Brief Symptom Inventory 18, administration, scoring, and procedure manual. San Antonio, TX: NCS Pearson; 2000.

36. IBM Corp. IBM SPSS Statistics for Windows, version 22.0. Armonk, NY: IBM; 2013.

37. Forero CG, Maydeu-Olivares A. Estimation of IRT graded response models: limited versus full information methods. Psychol Methods 2009;14:275-299.

38. Joreskog KG, Sorbom D. LISREL-VI user's guide, 3rd ed. Mooresville, IN: Scientific Software; 1984

39. Heywood HB. On finite sequences of real numbers. In: Proceedings of the Royal Society of London. Series A, containing papers of a mathematical and physical character. Vol. 134. London: Royal Society; 1931. p 486-501.

40. McDonald RP. Factor analysis and related methods. Hillsdale NJ: Erlbaum; 1985.

41. Felgoise S, Zaccheo V, Duff J, Simmons Z. Verbal communication impacts quality of life in patients with amyotrophic lateral sclerosis. Amyotroph Lateral Scler Frontotemporal Degener 2016;17: 179-183.

42. Mehta P, Anteo V, Kaye W, Sanchez M, Williamson D, Bryan L, et al. Prevalence of amyotrophic lateral sclerosis-United States, 2010-2011. MMWR Morbid Mortal Wkly Rep 2014;63.

43. National Institute of Neurological Disorders and Stroke. Amyotrophic lateral sclerosis (ALS) fact sheet. 2018. Available at https://www.ninds.nih.gov/ Disorders/Patient-Caregiver-Education/Fact-Sheets/AmyotrophicLateral-Sclerosis-ALS-Fact-Sheet. Accessed January 29, 2018.

44. Stephens HE, Green B, Bremer B, Felgoise S, Walsh S, Simmons Z. A pilot study to evaluate the reliability of administering the ALS-specific quality of life instrument-revised (ALSSQOL-R) in a self-administered format vs. interview based format. Amyotroph Lateral Scler 2007;8:103-104.

45. Schaefer M, Olson, DH. Assessing intimacy: the PAIR inventory. J Marital Fam Ther 1981;1:47-60.

46. Moore KA, McCabe MP, Stockdale JE. Factor analysis of the Personal Assessment of Intimacy in Relationships Scale (PAIR): engagement communication and shared friendships. Sex Relation Ther 1998;13: 361-368. 